\title{
DE LA REALIDAD EXTENDIDA AL METAVERSO: UNA REFLEXIÓN CRÍTICA SOBRE LAS APORTACIONES A LA EDUCACIÓN
}

\author{
From Extended Reality to The Metaverse: A Critical \\ Reflection on Contributions to Education
}

Pablo Javier ORTEGA-RODRÍGUEZ

Universidad Autónoma de Madrid. España.

pabloj.ortega@eduticuam.es

bttps://orcid.org/0000-0002-1128-2360

Fecha de recepción: 03/12/2021

Fecha de aceptación: 12/01/2022

Fecha de publicación en línea: 01/03/2022

Cómo citar este artículo: Ortega-Rodríguez, P. J. (2022). De la realidad extendida al metaverso: una reflexión crítica sobre las aportaciones a la educación. Teoría de la Educación. Revista Interuniversitaria, 34(2), 189-208. https://doi.org/10.14201/teri.27864

\section{RESUMEN}

La realidad extendida, un concepto que abarca la realidad virtual, la realidad aumentada y la realidad mixta, ha experimentado un notable progreso en los últimos años y ha tenido un gran impacto en la comprensión de la educación. La investigación sobre la realidad extendida ha aportado beneficios en la mejora de la enseñanza y el aprendizaje, a partir de dos conceptos clave: el grado de inmersión y la sensación de presencia. Sin embargo, estas aportaciones necesitan una revisión desde una metodología crítica fundamentada para evitar caer en la tecnofilia sin evidencias científicas. El objetivo de este artículo es conocer y evaluar las aportaciones de la realidad extendida a la educación y los retos que plantea ante el desarrollo del metaverso. Para ello, en 
primer lugar, se conceptualiza cada tipo de realidad y se evalúan sus aplicaciones en el ámbito de la educación desde un punto de vista crítico. En segundo lugar, se realiza una argumentación fundamentada sobre las ventajas e inconvenientes de estas tecnologías en la práctica educativa. En tercer lugar, se describen los desafíos que plantea el metaverso en la educación. Este análisis muestra como la realidad extendida ha contribuido al desarrollo de la innovación y la mejora del proceso de enseñanza y aprendizaje, aunque se evidencia la falta de formación del profesorado para diseñar experiencias de aprendizaje basadas en la realidad extendida y una carencia de colaboración interdisciplinar entre los ámbitos de la tecnología y la pedagogía. Estos resultados influyen en el desarrollo del metaverso, un paso más en la evolución de la realidad extendida, que tendrá un gran impacto en el ámbito de la educación, lo cual sugiere la necesidad de diseñar un código ético, desarrollar una cultura y proteger la identidad de los usuarios.

Palabras clave: realidad extendida; metaverso; realidad virtual; realidad aumentada; realidad mixta; teoría de la educación; TIC; aprendizaje.

\section{ABSTRACT}

Extended reality, a concept that encompasses virtual reality, augmented reality and mixed reality, has seen remarkable progress in recent years and has had a great impact on the understanding of education. Research on extended reality has provided benefits in improving teaching and learning, based on two key concepts: the degree of immersion and the sense of presence. However, these contributions need a review from a critical grounded theory, to avoid falling into a technophilia without scientific evidence. This article aims to know and evaluate the contributions of extended reality to education and the educational challenges it poses before the development of the metaverse. For this reason, first, each type of reality is defined and its applications in the field of education are evaluated from a critical point of view. Secondly, a critical argument is posed about the advantages and disadvantages of these technologies in educational practice. Third, challenges posed by the metaverse in education are described. This analysis shows that extended reality has contributed to the development of innovation and improvement of the teaching and learning process, although there is a lack of teacher training to design learning experiences based on extended reality and a lack of interdisciplinary collaboration between the fields of technology and pedagogy. These results influence the development of the metaverse, one more step in the evolution of extended reality, which will have a great impact in the field of education. Findings suggest the need to design an ethical code, develop a culture and protect identity of users.

Keywords: extended reality; metaverse; virtual reality; augmented reality; mixed reality; education theory; ICT; learning. 


\section{INTRODUCCIÓN}

El ámbito educativo está percibiendo cómo los adelantos que se producen en el campo de las TIC (Tecnologías de la Información y la Comunicación) le proporcionan nuevas herramientas de aprendizaje, que permiten al alumnado aumentar sus conocimientos más allá de las barreras temporales y geográficas (Cabero, 2013; Cabero et al., 2015). La investigación ha demostrado que el impacto de la incorporación de las TIC a las prácticas educativas se concreta en pequeñas innovaciones educativas ad hoc a la metodología del profesorado, dependiendo de su formación y actitudes hacia la enseñanza y el aprendizaje (Area, 2010; Fernández Cruz et al., 2018). Las tecnologías emergentes están transformando los modos tradicionales de enseñanza, que se aproximan a los escenarios en línea (Adell, 1997; Area, 2020). A pesar de los avances realizados en las infraestructuras, los recursos telemáticos, la formación y la competencia digital del profesorado, la presencia de las TIC ha representado un cambio superficial de las prácticas de enseñanza y aprendizaje (Area \& Adell, 2021).

En la última década, el aprendizaje online se ha extendido al ámbito educativo, de modo que las investigaciones se han centrado en conocer las posibilidades de las tecnologías emergentes en los entornos virtuales (Boulton et al., 2018; MuñozCristóbal et al., 2017). Schroeder (1996, p. 25) define entorno virtual como "una pantalla generada por ordenador que permite a los usuarios tener la sensación de estar presentes en un entorno diferente al que realmente se encuentra e interactuar con el mismo". Entre las tendencias más utilizadas en los entornos virtuales, destaca el mobile learning, un enfoque centrado en el aprendizaje de conocimientos mediante el uso de teléfonos móviles (Yuen \& Yuen, 2008). Este enfoque favorece el desarrollo de cuatro tipos de aprendizaje: el aprendizaje individualizado, que permite a los estudiantes aprender a su propio ritmo, el aprendizaje situado, que consiste en el uso de dispositivos móviles para aprender en un contexto real, el aprendizaje colaborativo, para interactuar y comunicarse fácilmente con otros estudiantes, y el aprendizaje informal, que tiene lugar cuando los estudiantes aprenden fuera de clase a su propio ritmo (Cheon et al., 2012). El uso del teléfono móvil con fines educativos ha cobrado protagonismo en los últimos años (Briz-Ponce et al., 2017). La literatura científica se centra en el impacto del mobile learning en el rendimiento académico del alumnado (Crompton \& Burke, 2018).

La pandemia causada por la COVID-19 ha puesto de relieve el papel que juegan las tecnologías emergentes en la educación. Las más utilizadas con el móvil son la realidad virtual (RV), considerada la herramienta de aprendizaje del siglo XX1 (Rogers, 2019), la realidad aumentada (RA) (Arici et al., 2019) y la realidad mixta (RM) (Zhan et al., 2020), que conforman la realidad extendida (XR, extended reality) (Çöltekin et al., 2020a). Este es un concepto que hace referencia a la combinación de los entornos virtuales y reales, y las interacciones que existen con los usuarios (Dall’Acqua \& Gironacci, 2019). Los avances en la realidad extendida permiten 
la validación de instrumentos que miden el grado de sensación de presencia que proporcionan los entornos virtuales, que abre nuevas posibilidades en la educación (Gandolfi et al., 2020).

Desde un punto de vista teórico, es preciso conocer las aportaciones que ofrece la realidad extendida, que puede ser un remedio, lo cual contribuye a la difusión de información, o algo perjudicial, que puede sustituir al profesorado (Meirieu, 2022). Asimismo, es necesario reflexionar de manera crítica sobre las aportaciones que ofrece la realidad extendida a la educación ante el desarrollo del metaverso, que constituye un reto al que debe enfrentarse la teoría de la educación.

El objetivo general de este artículo es conocer y evaluar las aportaciones de la realidad extendida (la realidad virtual, la realidad aumentada y la realidad mixta) a la educación y los retos educativos que plantea de cara al desarrollo del metaverso. Desde una metodología crítica fundamentada, es preciso conocer, en primer lugar, el concepto de cada tecnología y sus posibilidades en el ámbito de la educación desde un punto de vista crítico, evitando caer en la denominada tecnofilia, o entusiasmo sin evidencia científica hacia las posibilidades de las nuevas tecnologías. En segundo lugar, se reflexiona sobre las ventajas e inconvenientes de estas tecnologías en la práctica educativa. En tercer lugar, se describen los retos que plantea el metaverso en la educación del futuro, que abre nuevas líneas de investigación.

\section{Metodología}

Este trabajo se enmarca en una metodología crítica fundamentada, que parte del paradigma socio-crítico, basado en el análisis crítico de la realidad para transformar las prácticas educativas (Bisquerra, 2009). Esta metodología permite reflexionar sobre las aplicaciones, los pros y los contras de cada tipo de realidad a fin de aportar conocimientos a los investigadores y los profesionales de la educación y aplicarlos en el ámbito educativo (McMillan \& Schumacher, 2008). Se ha realizado un análisis de contenido sobre las referencias bibliográficas, con el programa ATLAS.ti, que ha permitido identificar cuatro categorías: el concepto de cada tipo de realidad, su aplicación en el ámbito educativo, las ventajas y los inconvenientes.

\section{Resultados}

\subsection{Concepto y aportaciones de la realidad virtual a la educación}

La realidad virtual (RV) es un entorno simulado, construido en formato digital, que cuenta con efectos visuales y sonoros para hacer creer a los usuarios que se encuentran en un ambiente que existe realmente. Puede aplicarse en el área educativa, sumergiendo al alumnado en un espacio creado para hacerle sentir distintas emociones, por ejemplo, pasear por las ciudades del antiguo Egipto (Gómez-García et al., 2020). Comprende dos conceptos clave: inmersión y presencia. El primero 
describe la experiencia resultante de usar la tecnología, que nos introduce en un ámbito imaginario y funciona intercambiando información sensorial de la realidad con otra, generada digitalmente, como imágenes y sonidos (Ott \& Freina, 2015). El segundo se refiere a la reacción subjetiva que experimentan los usuarios al estar inmersos en un entorno virtual, de modo que el cerebro se comporta de manera similar a encontrarse en el mundo real (Slater, 2003).

La literatura científica pone de relieve el uso de la realidad virtual en la educación y sus posibilidades en el proceso de enseñanza-aprendizaje. Los laboratorios en realidad virtual para enseñar contenidos relacionados con las áreas STEM (Science, Technology, Engineering and Mathematics) permiten llevar a cabo simulaciones de experimentos mediante objetos creados en 3D (Potkonjak et al., 2016). Son pocos los estudios que han diseñado aplicaciones de realidad virtual, basándose en una teoría de aprendizaje específica, y han investigado sobre cómo se puede integrar en el currículum (Radianti et al., 2020).

Diferentes investigaciones demuestran que las situaciones de realidad virtual producen desde sentimientos leves de inseguridad hasta otros más importantes de mareos (Jensen y Konradsen, 2017), que ponen de relieve la importancia de diseñar situaciones que minimicen las molestias físicas. El estudio de Krokos et al. (2019) muestra que el alumnado retiene más información y aplica mejor lo que ha aprendido tras haber participado en experiencias educativas con realidad virtual.

\subsubsection{Ventajas e inconvenientes de la realidad virtual}

Las ventajas de la RV residen en su capacidad para presenciar una situación. El alumnado siente una mayor sensación de presencia cuando se ve inmerso en una situación de realidad virtual con una HMD (Head-Mounted Display) o dispositivo de visualización similar a un casco, pero aprende menos en comparación con otra situación de baja inmersión en un ordenador (Makransky et al., 2017). Los inconvenientes de la RV son las dificultades técnicas que conlleva su uso y el alto coste del equipamiento necesario para llevar a cabo experiencias con alto nivel de inmersión (Duncan et al., 2012), que han dificultado su implementación en la práctica educativa. En este sentido, los pros relacionados con la realidad virtual en cuanto a la sensación de emociones en el alumnado no se han consolidado en la educación debido a la sofisticación y la inversión que exigen los dispositivos necesarios para su implementación en las aulas.

\subsection{Concepto y aportaciones de la realidad aumentada a la educación}

Thomas P. Caudell, investigador y científico, acuñó el término "realidad aumentada" (RA) en 1992 para definir una tecnología que permite aumentar el campo visual mediante la visualización frontal (Caudell \& Mizell, 1992; Lee, 2012). La realidad aumentada es una tecnología que permite a los usuarios ver una realidad 
complementada a través de objetos virtuales superpuestos sobre el mundo real (Azuma, 1997). Es un entorno que incluye elementos de dos mundos (real y virtual), mantiene interacción en directo y puede mostrarse en tres dimensiones (GómezGarcía \& Palomo, 2016, p. 141). A partir del año 2010, el número de investigaciones sobre la aplicación de la RA en la educación ha aumentado de manera notable debido a la integración de los sistemas de RA en los smartphones y las tablets, que destacan entre las tecnologías emergentes (Garzón et al., 2019).

La aplicación de la RA en la educación permite al profesorado y al alumnado hacer anotaciones en el mundo real, interactuar con objetos reales y virtuales a la vez, y realizar prácticas con objetos virtuales en una situación real sin riesgos, por ejemplo, la disección de animales (Gómez-García \& Palomo, 2016). En este sentido, uno de los ámbitos en los que la RA tiene mayor impacto es el ámbito STEM, en el que se utilizan herramientas de exploración basadas en la RA, junto a actividades de investigación y simulación (Ibáñez \& Delgado-Kloos, 2018). En el área de Matemáticas, el uso de la RA en la práctica educativa tiene un efecto significativo en la comprensión de conceptos (Boaler et al., 2016), la habilidad para resolver problemas (Sollervall, 2012) y el rendimiento académico (Demitriadou et al., 2020). Una de las modalidades de RA más común en el ámbito educativo es la geolocalización GPS, una tecnología que permite conocer la posición geográfica en cualquier sitio del mundo, acceder a mapas por geo-posicionamiento y a información en tiempo real (Brazuelo, 2015). La geolocalización y la realidad aumentada contribuyen a la realización de actividades de exploración del entorno de forma autónoma y de conceptos geográficos (Arango-López et al., 2019; Fombona \& Vázquez-Cano, 2017).

\subsubsection{Ventajas e inconvenientes de la realidad aumentada}

Las ventajas de la RA en la educación estriban en su capacidad para aumentar la participación del alumnado en las actividades con tecnologías inmersivas y mejorar la experiencia de aprendizaje (Suh \& Prophet, 2018). El uso de la RA en la práctica educativa tiene un efecto moderado en los resultados académicos del alumnado (Garzón \& Acevedo, 2019). Los inconvenientes de la RA tienen que ver con su complejidad, que pone de manifiesto la necesidad de formación específica del profesorado a fin de crear contenidos que mejoren el proceso de enseñanzaaprendizaje (Pellas et al., 2018). La RA plantea retos en la práctica educativa para mejorar el proceso de enseñanza-aprendizaje: la falta de formación y experiencia del profesorado, la necesidad de más tiempo de clase para perfeccionar su uso y su inadecuación en aulas con altas ratios (Ackayir \& Ackayir, 2017). La RA supone un paso más hacia la combinación de la RV y el mundo físico, que contribuye a la motivación del alumnado, aunque existen carencias formativas en los planes de estudios universitarios sobre su implementación en la práctica educativa. 


\subsection{Concepto y aportaciones de la realidad mixta a la educación}

Milgram y Kishino (1994), dos investigadores pioneros en tecnología explicaron las posibilidades de la RM en la mejora del aprendizaje "en cualquier lugar entre los extremos del continuo de virtualidad" (p. 1322), en el que los contextos virtuales se extienden de entornos reales a virtuales mediante una tecnología que puede "aumentar" la experiencia del usuario. En este sentido, acuñaron el término "realityvirtuality continuum" (véase la Figura 1), para designar la evolución de la RM.

FIGURA 1

EVOLUCIÓN DE LA REALIDAD MIXTA

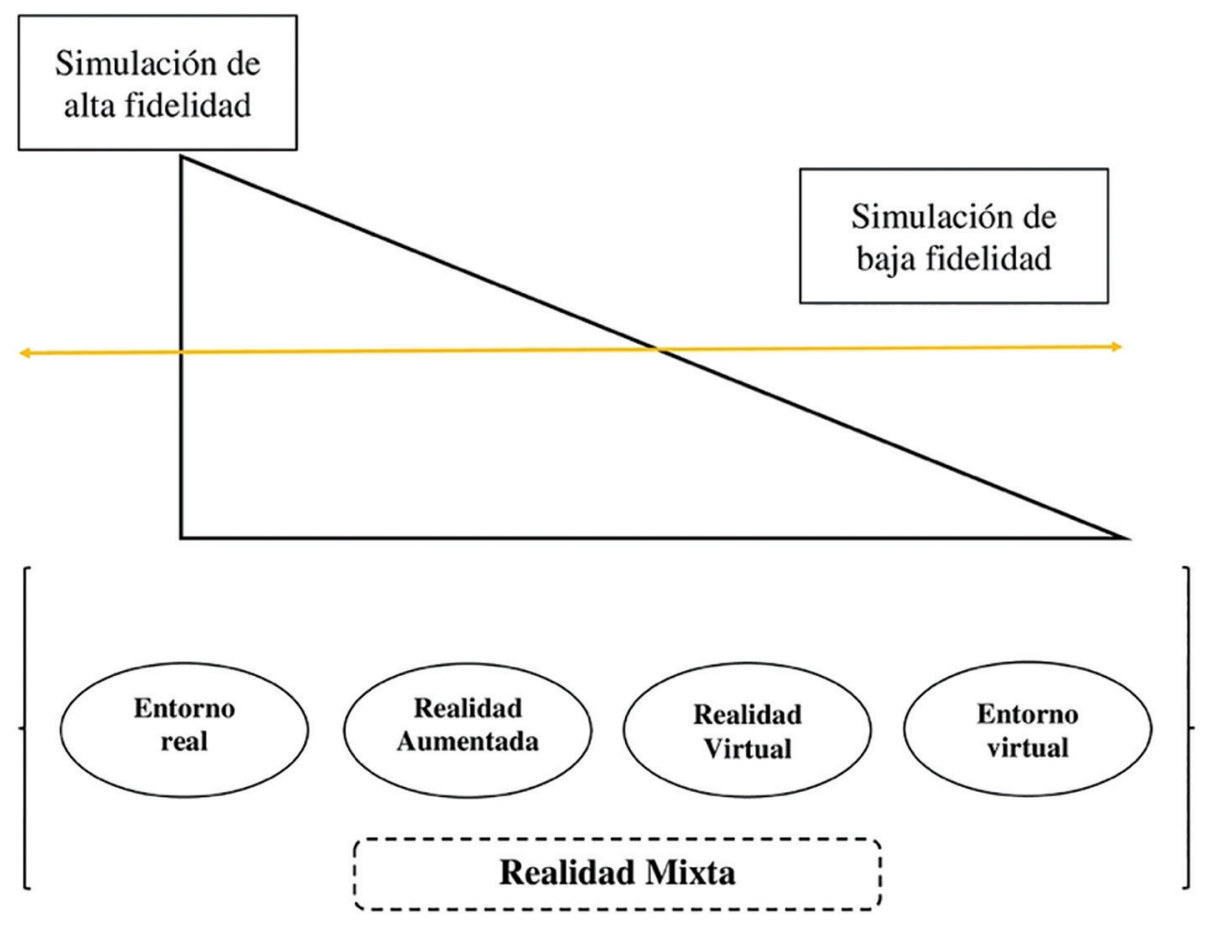

Fuente: Traducido de Pellas et al. (2019)

La RM es una combinación de la RA y la RV, que ofrece la posibilidad de interactuar físicamente con objetos virtuales en el mundo real (Yusoff et al., 2011). Se puede utilizar para el desarrollo de entornos "aumentados" dentro de otros pertenecientes al mundo real, en los que los usuarios pueden agregar o integrar información virtual dentro de ella (Tamura et al., 2001). En los entornos de RM, los 
datos se procesan a través de varios dispositivos de entrada diferentes, como gafas inteligentes, tabletas, sensores o computadoras personales (PC), que se combinan con dispositivos de salida, como proyectores, paredes interactivas o monitores de PC que muestran los resultados del procesamiento. Todos los dispositivos de entrada y salida deben integrarse dentro de un entorno físico que mejore la sensación de "presencia" del usuario (Ponto et al., 2006). La principal diferencia entre la RA y la RM estriba en que la primera permite la superposición de un objeto virtual en el mundo real, mientras que la segunda es un medio que incorpora un conjunto de elementos virtuales y reales a través de un continuo de la realidad (Lindgren \& Johnson-Glenberg, 2013). La investigación ha demostrado que el uso de la RM resulta eficaz en el proceso de enseñanza-aprendizaje (Ke et al., 2016; Tang et al., 2020).

Los principales retos que plantean la RV, la RA y la RM son la personalización del aprendizaje y la inteligencia educativa. La personalización del aprendizaje conlleva crear e implementar experiencias de aprendizaje basadas en las necesidades educativas y características del alumnado (conocimientos previos sobre un tema, intereses y estilos de aprendizaje). La inteligencia educativa es la aplicación de las nuevas tecnologías y los métodos inteligentes que mejoran el aprendizaje del alumnado (Kurilovas, 2016).

La aplicación de la RM en el ámbito educativo ha sido un tema estudiado en profundidad en muchas investigaciones (Johnson-Glenberg et al., 2014; Tscholl \& Lindgren, 2016). La aplicación de la realidad mixta en la educación ha dado lugar a un enfoque denominado MRLE (Mixed Reality Learning Environment) o entorno de aprendizaje basado en la realidad mixta (Chang et al., 2010). Una de las aplicaciones del MRLE que ha tenido mayor impacto en el aprendizaje es el uso de los móviles en el área de ciencias, mediante el diseño de laboratorios en realidad mixta, que permiten a los usuarios enfocar las pruebas del laboratorio, aumentar los gráficos, modificarlos y realizar experimentos (Frank \& Kapila, 2017). También se ha utilizado en el área de astronomía para enseñar conocimientos sobre el espacio exterior (Lindgren et al., 2016).

La motivación del alumnado es un factor importante en el rendimiento académico (Trowler, 2010). Tanto el uso de la RV como de la RM tiene más impacto en la motivación del alumnado que los métodos tradicionales, lo cual influye en la mejora del rendimiento académico (Allcoat et al., 2021; Leonard \& Fitzgerald, 2018). El uso de la RM en la práctica educativa mejora el rendimiento académico del alumnado, en particular aquel que tiene baja habilidades espaciales (Weng et al., 2018).

\subsubsection{Ventajas e inconvenientes de la realidad mixta}

Las ventajas de la RM guardan relación con sus múltiples aplicaciones desde un punto de vista metodológico, en tanto que combina las posibilidades de la RV y RA en un mismo enfoque, dando lugar al diseño y desarrollo de experiencias de aprendizaje que otorgan relevancia, interés y significancia al aprendizaje del 
alumnado. Uno de los enfoques en los que la RM destaca es el serious game, que consiste en un juego digital cuyo objetivo es entretener y alcanzar, al menos, un objetivo educativo, promoviendo la resolución de problemas (Dörner et al., 2016). En este sentido, los serious games basados en la RM contribuyen a que los alumnos resuelvan un problema significativo de forma colaborativa, aprovechando las posibilidades que ofrecen ambas realidades (Lee et al., 2016). Los inconvenientes derivados de la aplicación de la RM en la educación tienen que ver con la carencia de colaboración interdisciplinar entre la investigación tecnológica de los soportes utilizados en la RM (por ejemplo: las gafas inteligentes) y la pedagógica sobre su aplicación en la práctica educativa. La creación de experiencias basadas en la RM que resulten eficientes sólo puede lograrse mediante la colaboración interdisciplinar, consolidando y aceptando los avances de diferentes disciplinas, como la tecnología y la educación, de modo que el avance de las disciplinas por separado conlleva el riesgo de hacer suposiciones unidireccionales sobre el uso de las tecnologías (Çoltekin et al., 2020b). En este sentido, el avance en el conocimiento sobre estas nuevas tecnologías impulsa enfoques interdisciplinares con un gran potencial que permiten todo tipo de metodologías y aplicaciones (Prendes \& Cerdán, 2021).

La revisión de la literatura ha demostrado que existe una carencia de estudios sobre el uso de la RA y la RV con fines educativos debido a tres factores: 1. El acceso a estas tecnologías mediante los smartphones, cuyo uso no ha llegado a consolidarse en el proceso de enseñanza-aprendizaje, 2. La falta de contenidos sobre la utilización de estas tecnologías en los centros educativos y 3. La brecha digital en países y escuelas (Maas \& Hughes, 2020). Las experiencias de RM en el metaverso requerirán de interacción verbal con objetos virtuales y recibir un feedback o retroalimentación, de modo que la interacción de voz es el reto principal para pasar de la RM al desarrollo del metaverso en la educación (Siyaev \& Jo, 2021). Para ello, es preciso perfeccionar el desarrollo de las gafas empleadas en la RM, que funcionan con comandos de voz estáticos y predefinidos (Eschen et al., 2018), e incluir otros flexibles que se adapten al feedback de los usuarios.

\subsection{Concepto y aportaciones del metaverso a la educación}

Mark Zuckerberg anunció en octubre de 2021 la llegada del metaverso en un plazo de 5 a 10 años, definiéndolo como una realidad digital alternativa en la que la gente trabaja, juega y socializa (Gonzalo, 2021). Desde entonces, han proliferado artículos de opinión sobre las posibles consecuencias del metaverso en la sociedad y su impacto en el e-learning, poniendo de relieve la importancia y vigencia del tema. Para conocer los retos que plantea el metaverso en los escenarios digitales del futuro, procede partir del concepto y las experiencias que se han aproximado a esta nueva realidad, publicadas en revistas científicas. Los conceptos que aluden al metaverso son "mundo espejo" o "internet espacial", de modo que puede entenderse como "una realidad física virtualmente mejorada" (Ribeiro, 2021). 
El metaverso es un acrónimo formado por el prefijo "meta" (más allá) y la contracción del término universo -"verso"-, que se refiere a un mundo virtual en 3D que es inmersivo, interactivo y colaborativo. Constituye una nueva dimensión en Internet, con gran impacto en la educación del futuro (Rodríguez, 2021). Fue el novelista americano Neal Stephenson (1992) quien utilizó este término por primera vez en su novela Snow Crash para referirse a una nueva versión de Internet. Esto permite que diferentes miembros de un grupo puedan trabajar juntos en un espacio virtual mediante avatares que interactúan cara a cara y crean objetos tridimensionales que representan ideas, valores o sentimientos (Davis et al., 2009).

El metaverso reúne una serie de características que superan a la realidad extendida (Ball, 2020; Martín-Ramallal \& Merchán-Murillo, 2019):

- Es persistente. No se reinicia ni se pausa, sino que continúa indefinidamente.

- Favorece la proactividad de los usuarios, que toman el control, adoptan decisiones y se anticipan a posibles acontecimientos.

- Es sincrónico y existe en tiempo real.

- No tiene límites para la simultaneidad de usuarios en una actividad.

- Brinda una experiencia que abarca el mundo digital y el real, la relación entre redes privadas y públicas.

- Ofrece interoperabilidad de datos y elementos digitales, por ejemplo, un objeto virtual de un videojuego puede ser regalado a un amigo a través de Facebook.

Una de las posibilidades educativas del metaverso está relacionada con la creación de museos, que albergan exposiciones de temas especiales y proporcionan contenidos experienciales a los visitantes (Choi \& Kim, 2017). Otras guardan relación con el diseño de experiencias de aprendizaje basadas en el metaverso, que exigen la creación de un avatar, un alter ego del usuario que puede interactuar con otros avatares en un mundo virtual (Bailenson \& Yee, 2005). El Informe Horizon Report 2021 destaca el uso de avatares para practicar un idioma en un entorno virtual. El avatar está conectado con un chatbot, que ofrece respuestas a las dudas del alumnado (Pelletier et al., 2021). Un estudio pionero evaluó la percepción social de las personas a partir de la creación de dos avatares, uno tiene la misma apariencia facial que los usuarios y el otro, la misma expresión de sus gestos. Los resultados muestran que los participantes no se identificaban con la apariencia de su avatar, mientras que sí se reconocieron en los avatares que realizaban los mismos gestos que ellos. Por tanto, este realismo conductual juega un papel importante en el diseño de avatares para la implementación del metaverso (Park et al., 2021).

\subsubsection{Retos del metaverso en la educación}

El principal reto estriba en pasar de un entorno en realidad virtual, sofisticado, pero independiente, a una red integrada de mundos virtuales en 3D o metaverso, que pone de relieve la necesidad de avanzar en el conocimiento sobre cuatro aspectos 
que tienen un impacto en la educación (Dionisio et al., 2013): 1. Realismo (hacer sentir a los usuarios que están inmersos en una dimensión alternativa), 2. Ubicuidad (establecer el acceso al sistema a través de los dispositivos digitales y mantener la identidad virtual de los usuarios dentro del sistema), 3. Interoperabilidad (permitir la creación y movimiento de objetos en 3D fuera del sistema), y 4. Escalabilidad (permitir un uso eficiente del sistema con cantidades masivas de usuarios conectados a la vez). Desde un punto de vista práctico, las primeras experiencias que se han aproximado al metaverso se han centrado en la comprensión escrita y oral de idiomas (Garrido-Íñigo \& Rodríguez-Moreno, 2013).

El metaverso ofrece muchas posibilidades educativas relacionadas con la adquisición de conocimientos culturales (Zheng, 2020). En este sentido, uno de los conceptos clave en el desarrollo del metaverso es la cultura, que se refiere a los antecedentes y experiencias de las personas que forman parte de esta dimensión (Han, 2015) y abarca dos conceptos relacionados: quiénes somos y cómo vivimos (Wang, 2001). Castells (2005, p. 405) afirma que es precisa una cultura en la sociedad del conocimiento, a partir del establecimiento de ciertas normas. El autor señala que esta cultura forma parte de un sistema de virtualidad real, en el que la misma realidad (la existencia de las personas) es sumergida en un escenario de imágenes virtuales, en el que las apariencias no están solamente en la pantalla a través de la que se comunica la experiencia, sino que se convierten en la experiencia. En este sentido, resulta fundamental construir una cultura o conjunto de normas y valores de base para comunicarse, trabajar y ser en el metaverso, una realidad alternativa en la que las fronteras entre lo físico y lo virtual resultan aún difusas y necesitan ser delimitadas con precisión para salvaguardar la identidad de los usuarios. En este sentido, Smithson (2022) sugiere la necesidad de un código ético para desarrollar la cultura del metaverso, que se sustenta en cinco aspectos:

1. La transparencia de conocimientos y opiniones entre los consumidores y los creadores de productos basados en el metaverso contribuyen a la mejora de su funcionamiento.

2. Una escala de valores que favorezca la convivencia y las relaciones entre los usuarios.

3. El principio de inclusión es fundamental. El metaverso debe ser accesible y fácil de utilizar para todas las personas.

4. Los avatares (o alter ego) son personas reales, de modo que las actitudes de acoso y fraudulentas serán sancionadas con la eliminación temporal de los usuarios en este espacio.

5. La ética es la piedra angular, que se traduce en el diseño de programas y experiencias de aprendizaje que aseguren la confidencialidad de los consumidores.

\section{ConClusiones}

La pandemia originada por la COVID-19 supuso el traslado de la docencia a las plataformas digitales, que marca un punto de inflexión sobre la implementación de 
las tecnologías emergentes en la educación del futuro, en la que la realidad extendida y el metaverso destacan por su relevancia, interés e impacto en el proceso de enseñanza-aprendizaje. Por ello, el objetivo fundamental de este estudio era conocer y evaluar las aportaciones científicas de la realidad extendida (la realidad virtual, la realidad aumentada y la realidad mixta) al ámbito educativo, así como los retos que plantea el metaverso en la educación del futuro.

En cuanto al concepto de los tres tipos de realidades que conforman la realidad extendida, la RV incorpora efectos visuales y sonoros para hacer creer a los usuarios que están en un ambiente que existe en la realidad. El objetivo fundamental de esta realidad es abordar la inmersión en un ámbito virtual sin renunciar a la presencia ni a la reacción subjetiva de los usuarios. Ambos conceptos, inmersión y presencia, han guiado los estudios posteriores sobre la realidad aumentada, que brinda la posibilidad de interactuar físicamente con objetos virtuales en el mundo real. La RA se ha visto reforzada por los smartphones y las tablets, que han facilitado su desarrollo en la educación. En un intento por aumentar el grado de inmersión y presencia, surge la realidad mixta, que incorpora elementos virtuales y reales a través de un continuo de la realidad y constituye un antecedente importante para el desarrolló del metaverso, un mundo virtual en 3D que requiere la creación de un avatar para interactuar con otros avatares en un mundo virtual.

En lo que concierne a la aplicación en el ámbito educativo, la RV se ha centrado en el diseño de laboratorios sobre las áreas STEM para llevar a cabo simulaciones de experimentos sin riesgo alguno. La aplicación de la RA se ha extendido al área de las matemáticas, para la explicación de conceptos, y de la geografía, para la exploración de entornos geográficos mediante la geolocalización. La implementación de la realidad mixta ha supuesto el perfeccionamiento de las pruebas de laboratorio en el área de ciencias, mediante la modificación y el aumento de gráficos, así como la enseñanza de conceptos sobre el espacio exterior en el área de astronomía. Asimismo, ha supuesto un avance notable en el desarrollo de nuevas propuestas metodológicas, como los serious games.

En lo tocante a las ventajas e inconvenientes de la realidad extendida, la realidad virtual ofrece mayor sensación de inmersión y presencia que las metodologías tradicionales, pero el coste del equipamiento necesario para desarrollar experiencias de calidad en los centros educativos es alto. La realidad aumentada favorece la motivación del alumnado hacia el aprendizaje, aunque el profesorado no cuenta con la formación necesaria para implementarla en la práctica educativa. Por su parte, la realidad mixta promueve el desarrollo de estrategias metodológicas diversas, aunque los avances en los campos de la tecnología y la pedagogía han discurrido por cauces paralelos, sin que exista una colaboración interdisciplinar para avanzar en el conocimiento sobre las posibilidades educativas de la RM en la educación. 
Cuatro son los grandes retos que afronta la educación para desarrollar la realidad extendida en el ámbito educativo (Fernández, 2021; López- Rupérez, 2020; NEPT., 2017):

- La alta inversión que han de realizar los centros para poner en práctica la RV y la RA, que permiten crear nuevas experiencias de aprendizaje en laboratorios digitales.

- La personalización de la formación, que tiene en cuenta el punto de partida del alumnado, de modo que el ritmo de aprendizaje y el enfoque de la enseñanza están organizados según las necesidades de cada estudiante.

- La integración de metodologías activas y las tecnologías emergentes, que implica el desarrollo de metodologías, como la clase invertida o flipped classroom, en la que los alumnos preparan el temario antes de una clase y dedican el tiempo en el aula a poner en práctica tales contenidos.

- La búsqueda de un aprendizaje significativo, que no conciba las tecnologías emergentes sólo como un fin en sí mismo, sino también como herramientas al servicio del profesorado y del alumnado para lograr una mejora del proceso de enseñanza-aprendizaje.

Estos desafíos influyen en el desarrollo del metaverso, que está llamado a ser una revolución en los ámbitos de las nuevas tecnologías, la comunicación y la educación. En esta nueva realidad es preciso diseñar un avatar, que represente al usuario e interactúa con otros avatares para comunicarse, trabajar y colaborar, lo cual abre muchas posibilidades en el e-learning, trascendiendo las barreras temporales y geográficas. Existen tres aspectos que cobran importancia en las futuras investigaciones:

1. El desarrollo del metaverso puede difuminar las fronteras entre el mundo real y el virtual, que sugiere la necesidad de diseñar y desarrollar un código ético que sirva de guía para desenvolverse de manera segura. No se trata de que esta realidad "alternativa" suplante a la realidad en sí misma, sino que se perciba como un nuevo continuo de la realidad virtualmente mejorada, lo cual abre nuevas posibilidades en el proceso de enseñanza-aprendizaje.

2. Este código ético favorece el desarrollo de una cultura, que consiste en el diseño e implantación de normas que cobran un sentido en el metaverso y se consolidan con la experiencia. Dos aspectos fundamentales de este código ético son el principio de inclusión y los valores, que favorecen el desarrollo de las relaciones entre los usuarios.

3. La protección de la identidad de los usuarios. La creación de un avatar implica definir un alter ego, que reúne una serie de rasgos físicos y/o de personalidad que nos definen como individuos en el metaverso y nos diferencian del resto de avatares. Esta situación exige la necesidad de garantizar la confidencialidad de los datos personales. 
PABLO JAVIER ORTEGA-RODRÍGUEZ

DE LA REALIDAD EXTENDIDA AL METAVERSO:

UNA REFLEXIÓN CRÍTICA SOBRE LAS APORTACIONES A LA EDUCACIÓN

A modo de conclusión, se puede afirmar que el traspaso de la realidad extendida al metaverso en el e-learning conlleva un planteamiento diferente, en tanto que la inmersión y la presencia constituían un medio para mejorar el proceso de enseñanza-aprendizaje, mientras que en el metaverso representan un fin en sí mismo, en una realidad virtualmente mejorada. Resulta necesario una mayor colaboración interdisciplinar entre los campos de la tecnología y la investigación en educación a fin de que la implantación del metaverso en el e-learning conduzca a una mejora de la enseñanza y el aprendizaje.

\section{REFERENCIAS BIBLIOGRÁFICAS}

Adell, J. (1997). Tendencias en educación en la sociedad de las tecnologías de la información. Edutec. Revista Electrónica de Tecnología Educativa, (7), a007. https://doi.org/10.21556/ edutec.1997.7.570

Akçayır, M., \& Akçayır, G. (2017). Advantages and challenges associated with augmented reality for education: A systematic review of the literature. Educational Research Review, 20(1), 1-11. https://doi.org/10.1016/j.edurev.2016.11.002

Allcoat, D., Hatchard, T., Azmat, F., Stansfield, K., Watson, D., \& von Mühlenen, A. (2021). Education in the digital age: learning experience in virtual and mixed realities. Journal of Educational Computing Research, 59(5), 795-816. https://doi.org/10.1177/0735633120985120

Arango-López, J., Cerón, C. C., Collazos, C.A., Gutiérrez, F. L., \& Moreira, F. (2019). Creando: Tool for creating pervasive games to increase the learning motivation in higher education students. Telematics and Informatics, (38), 62-73. https://doi.org/10.1016/j.tele.2018.08.005

Area, M. (2010). El proceso de integración y uso pedagógico de las TIC en los centros educativos. Un estudio de casos. Revista de Educación, (352), 77-97.

Area, M. (2020). Escuela Digital. Los materiales didácticos en la Red. Graó.

Area, M., \& Adell, J. (2021). Tecnologías digitales y cambio educativo. Una aproximación crítica. REICE. Revista Iberoamericana sobre Calidad, Eficacia y Cambio en Educación, 19(4), 83-96. https://doi.org/10.15366/reice2021.19.4.005

Arici, F., Yildirim, P., Caliklar, S., \& Yilmaz, R. M. (2019). Research trends in the use of augmented reality in science education: Content and bibliometric mapping analysis. Computers E Education, 142(103647), 1-44. https://doi.org/10.1016/j.compedu.2019.103647

Azuma, R. (1997). A survey of augmented reality. Presence: Teleoperators and Virtual Environments, 6(4), 355-385. https://doi.org/10.1162/pres.1997.6.4.355

Bailenson, J. N., \& Yee, N. (2005). Digital chameleons: Automatic assimilation of nonverbal gestures in immersive virtual environments. Psychological Science, 16(10), 814-819. https://doi.org/10.1111/j.1467-9280.2005.01619.x

Ball, M. (14 de enero de 2020). The Metaverse: what it is, where to find it, and who will build it. https://bit.ly/34U1SW5

Bisquerra, R. (2009). Metodología de la investigación educativa. La Muralla.

Boaler, J., Chen, L., Williams, C., \& Cordero, M. (2016). Seeing as understanding: The importance of visual mathematics for our brain and learning. Journal of Applied \& Computational Mathematics, 5(5), 1-6. https://doi.org/10.4172/2168-9679.1000325 
Boulton, C. A., Kent, C., \& Williams, H. (2018). Virtual learning environment engagement and learning outcomes at a "bricks-and-mortar" university. Computers E Education, (126), 129-142. https://doi.org/10.1016/j.compedu.2018.06.031

Brazuelo, F. (2015). Recursos móviles y realidad aumentada. En M. L. Cacheiro, C. Sánchez \& J. M. González (Coords.), Recursos tecnológicos en contextos educativos (pp. 399-432). UNED.

Briz-Ponce, L., Pereira, A., Carvalho, L., Juanes-Méndez, J. A., \& García-Peñalvo, F. J. (2017). Learning with mobile technologies-Students' behaviour. Computers in Human Behavior, 72, 612-620. https://doi.org/10.1016/j.chb.2016.05.027

Cabero, J. (2013). La formación virtual en el nuevo entramado 2.0: el e-learning 2.0. En J. I. Aguaded \& J. Cabero (Coords.), Tecnologías y medios para la educación en la e-sociedad (pp. 23-52). Alianza.

Cabero, J., Barroso, J., \& Llorente M. C. (2015). Tecnología educativa: historia, concepto y bases conceptuales. En J. Cabero \& J. Barroso (Coords.), Nuevos retos en tecnología educativa (pp. 19-39). Síntesis.

Castells, M. (2005). La era de la información: economía, sociedad y cultura. Volumen 1. Siglo XXI editores.

Caudell, T. P., \& Mizell, D. W. (1992). Augmented reality: an application of heads-up display technology to manual manufacturing processes. Proceedings of the Twenty-Fifth Hawaii International Conference on System Sciences, 2, 659-669. https://doi.org/10.1109/ HICSS.1992.183317

Chang, C. W., Lee, J. H., Wang, C. Y., \& Chen, G. D. (2010). Improving the authentic learning experience by integrating robots into the mixed-reality environment. Computers $\mathcal{E}$ Education, 55(4), 1572-1578. https://doi.org/10.1016/j.compedu.2010.06.023

Cheon, J., Lee, S., Crooks, S. M., \& Song, J. (2012). An investigation of mobile learning readiness in higher education based on theory of planned behavior. Computers $\varepsilon$ Education, 59(3), 1054-1064. https://doi.org/10.1016/j.compedu.2012.04.015

Choi, H., \& Kim, S. (2017). A content service deployment plan for metaverse museum exhibitions-Centering on the combination of beacons and HMDs. International Journal of Information Management, 37(1), 1519-1527. https://doi.org/10.1016/j.ijinfomgt.2016.04.017

Çöltekin, A., Griffin, A. L., Slingsby, A., Robinson, A. C., Christophe, S., Rautenbach, V., Chen, M., Pettit, C., \& Klippel, A. (2020a). Geospatial information visualization and extended reality displays. En Manual of Digital Earth (pp. 229-277). Springer.

Çoltekin, A., Lochhead, I., Madden, M., Christophe, S., Devaux, A., Pettit, C., Lock, O., Shukla, S., Herman, L., Stachon, Z., Kubícek, P., Snopkova, D., Bernardes, S., \& Hedley, N. (2020b). Extended Reality in spatial sciences: A review of research challenges and future directions. ISPRS International Journal of Geo-Information, 9(7), 1-29. https:// doi.org/10.3390/ijgi9070439

Crompton, H., \& Burke, D. (2018). The use of mobile learning in higher education: A systematic review. Computers \& Education, 123, 53-64. https://doi.org/10.1016/j.compedu.2018.04.007

Dall'Acqua, L., \& Gironacci, I. M. (2019). Political decision-making and security intelligence: Recent techniques and technological developments. IGI Global. 
PABLO JAVIER ORTEGA-RODRÍGUEZ

DE LA REALIDAD EXTENDIDA AL METAVERSO:

UNA REFLEXIÓN CRÍTICA SOBRE LAS APORTACIONES A LA EDUCACIÓN

Davis, A., Khazanchi, D., Murphy, J., Zigurs, I., \& Owens, D. (2009). Avatars, people, and virtual worlds: Foundations for research in metaverses. Journal of the Association for Information Systems, 10(2), 90-116. https://doi.org/0.17705/1jais.00183

Demitriadou, E., Stavroulia, K. E., \& Lanitis, A. (2020). Comparative evaluation of virtual and augmented reality for teaching mathematics in primary education. Education and Information Technologies, 25(1), 381-401. https://doi.org/10.1007/s10639-019-09973-5

Dionisio, J. D., Burns, W. G., \& Gilbert, R. (2013). 3D virtual worlds and the metaverse: Current status and future possibilities. AMC Computing Surveys, 45(3), 1-38. https://doi. org/10.1145/2480741.2480751

Dörner, R., Göbel, S., Effelsberg, W., \& Wiemeyer, J. (2016). Serious games. Foundations, concepts and practice. Springer.

Duncan, I., Miller, A., \& Jiang, S. (2012). A taxonomy of virtual worlds usage in education. British Journal of Educational Technology, 43(6), 949-964. https://doi.org/10.1111/j.14678535.2011.01263.x

Eschen, H., Kötter, T., Rodeck, R., Harnisch, M., \& Schüppstuhl, T. (2018). Augmented and Virtual Reality for inspection and maintenance processes in the aviation industry. Procedia Manufacturing, 19, 156-163. https://doi.org/10.1016/j.promfg.2018.01.022

Fernández, J. A. (20 de noviembre de 2021). Cinco asignaturas que debe aprobar la universidad que viene. El País. https://bit.ly/3Kli1En

Fernández Cruz, F. J., Fernández Díaz, M. J., \& Rodríguez Mantilla, J. M. (2018). El proceso de integración y uso pedagógico de las TIC en los centros educativos madrileños. Educación XX1, 21(2), 395-416. https://doi.org/10.5944/educxx1.17907

Fombona, J., \& Vázquez-Cano, E. (2017). Posibilidades de utilización de la Geolocalización y realidad aumentada en el ámbito educativo. Educación XX1, 20(2), 319-342. https:// doi.org/10.5944/educXX1.19046

Frank, J. A., \& Kapila, V. (2017). Mixed-reality learning environments: Integrating mobile interfaces with laboratory test-beds. Computers \& Education, 110(4), 88-104. https:// doi.org/10.1016/j.compedu.2017.02.009

Gandolfi, E., Kosko, K. W., \& Ferdig, R. E. (2020). Situating presence within extended reality for teacher training: Validation of the extended Reality Presence Scale (XRPS) in preservice teacher use of immersive 360 video. British Journal of Educational Technology, 52(2), 824-841. https://doi.org/10.1111/bjet.13058

Garzón, J., \& Acevedo, J. (2019). Meta-analysis of the impact of Augmented Reality on students' learning gains. Educational Research Review, 27(1), 244-260. https://doi.org/10.1016/j. edurev.2019.04.001

Garzón, J., Pavón, J., \& Baldiris, S. (2019). Systematic review and meta-analysis of augmented reality in educational settings. Virtual Reality, 23(1), 447-459. https://doi.org/10.1007/ s10055-019-00379-9

Garrido-Iñigo, P., \& Rodríguez-Moreno, F. (2013). The reality of virtual worlds: pros and cons of their application to foreign language teaching. Interactive Learning Environments, 23(4), 1-18. https://doi.org/10.1080/10494820.2013.788034 
Gómez-García, M., \& Palomo, R. (2016). Realidad aumentada y recursos móviles. En J. Sánchez, J. Ruiz \& M. Gómez García (Coords.), Tecnologías de la Comunicación y la Información aplicadas a la educación (pp. 131-149). Síntesis.

Gómez-García, M., Palomo, R., Guillén, F. D., \& Pérez, R. (2020). Aprendizaje con móviles. realidad virtual, aumentada y mixta. En E. Sánchez, E. Colomo \& J. Ruiz (Coords.), Tecnologías de la Información y la Comunicación en Contextos Educativos (pp. 141-152). Síntesis.

Gonzalo, M. (26 de octubre de 2021). Metaverso, ¿para cuándo? O cómo reconocerlo cuando llegue. https://bit.ly/3Gesijl

Han, H. C. (2015). Teaching visual learning through virtual world experiences: Why do we need a virtual world for art education? Art Education, 68(6), 22-27. https://doi.org/10. 1080/00043125.2015.11519344

Ibáñez, M. B., \& Delgado-Kloos, C. (2018). Augmented reality for STEM learning: A systematic review. Computers \& Education, 123, 109-123. https://doi.org/10.1016/j. compedu.2018.05.002

Jensen, L., \& Konradsen, F. (2017). A review of the use of virtual reality head-mounted displays in education and training. Education and Information Technologies, 23(4), 1515-1529. https://doi.org/10.1007/s10639-017-9676-0

Johnson-Glenberg, M. C., Birchfield, D. A., Tolentino, L., \& Koziupa, T. (2014). Collaborative embodied learning in mixed reality motion-capture environments: Two science studies. Journal of Educational Psychology, 106(1), 86-104. https://doi.org/10.1037/a0034008

Ke, F., Lee, S., \& Xu, X. (2016). Teaching training in a mixed-reality integrated learning environment. Computers in Human Behavior, 62, 212-220. https://doi.org/10.1016/j. chb.2016.03.094

Krokos, E., Plaisant, C., \& Varshney, A. (2019). Virtual memory palaces: immersion aids recall. Virtual Reality, (23), 1-15. https://doi.org/10.1007/s10055-018-0346-3

Kurilovas, E. (2016). Evaluation of quality and personalization of VR/AR/MR learning systems. Behaviour and Information Technology, 35(11), 998-1007. https://doi.org/10.1080/014 4929X.2016.1212929

Lee, K. (2012). Augmented reality in education and training. TechTrends, 56(2), 13-21. https:// doi.org/10.1007/s11528-012-0559-3

Lee, H., Parsons, D., Kwon, G., Kim, J., Petrova, K., JEong, W., \& Ryu, H. (2016). Cooperation begins: Encouraging critical thinking skills through cooperative reciprocity using a mobile learning game. Computers \& Education, 97, 97-115. https://doi.org/10.1016/j. compedu.2016.03.006

Leonard, S. N., \& Fitzgerald, R. N. (2018). Holographic learning: A mixed reality trial of Microsoft holoLens in an Australian secondary school. Research in Learning Technology, 26, 1-12. https://doi.org/10.25304/rlt.v26.2160

Lindgren, R., \& Johnson-Glenberg, M. (2013). Emboldened by embodiment: Six precepts for research on embodied learning and mixed reality. Educational Researcher, 42(8), 445-452. https://doi.org/10.3102/13189X13511661

Lindgren, R., Tscholl, M., Wang, S., \& Johnson, E. (2016). Enhancing learning and engagement through embodied interaction within a mixed reality simulation. Computers $\&$ Education, 95(1), 174-187. https://doi.org/10.1016/j.compedu.2016.1.1 
PABLO JAVIER ORTEGA-RODRÍGUEZ

DE LA REALIDAD EXTENDIDA AL METAVERSO:

UNA REFLEXIÓN CRÍTICA SOBRE LAS APORTACIONES A LA EDUCACIÓN

López-Rupérez, F. (2020). El currículo y la educación en el siglo XXI. La preparación del futuro y el enfoque por competencias. Narcea.

Maas, M. J., \& Hughes, J. M. (2020). Virtual, augmented and mixed reality in K-12 education: a review of the literature. Technology, Pedagogy and Education, 29(2), 231-249. https:// doi.org/10.1080/1475939X.2020.1737210

Makransky, G., Terkildsen, T. S., \& Mayer, R. E. (2017). Adding immersive virtual reality to a science lab simulation causes more presence but less learning. Learning \& Instruction, 60, 225-236. https://doi.org/10.1016/j.learninstruc.2017.12.007

Martín-Ramallal, P., \& Merchán-Murillo, A. (2019). Realidad virtual. Metaversos como herramienta para la teleformación. En P. Casas-Moreno, G. Paramio-Pérez \& V. B. Gómez Pablos (Eds.), Realidades educativas en la esfera digital: Sistemas, modelos y paradigmas de aprendizaje (pp. 15-38). Egregius Ediciones.

McMillan, J., \& Schumacher, S. (2008). Investigación educativa: una introducción conceptual. Pearson.

Meirieu, Ph. (2022). El futuro de la Pedagogía. Teoría de la Educación. Revista Interuniversitaria, 34(1), 69-81. https://doi.org/10.14201/teri.27128

Milgram, P., \& Kishino, A. (1994). Taxonomy of mixed reality visual displays. IEICE Transactions on Information and Systems, 12(12), 1321-1329.

Muñoz-Cristóbal, J. A., Gallego-Lema, V., Arribas-Cubero, H. F., Martínez-Monés, A., \& AsensioPérez, J. I. (2017). Using virtual learning environments in bricolage mode for orchestrating learning situations across physical and virtual spaces. Computers E Education, 109, 233-252. https://doi.org/10.1016/j.compedu.2017.03.004

NEPT. (2017). Reimagining the role of technology in education. U.S. Department of Education. https://tech.ed.gov.

Ott, M., \& Freina, L. (23 de abril de 2015). A literature review on immersive virtual reality in education: state of the art and perspectives [Presentación de paper]. Conference: eLearning and Software for Education, Bucarest, Rumanía. https://bit.ly/3DdqeFT

Park, S., Kim, S. P., \& Whang, M. (2021). Individual's social perception of virtual avatars embodied with their habitual facial expressions and facial appearance. Sensors, 21 (5986), 1-18. https://doi.org/10.3390/s21175986

Pellas, N., Fotaris, P., Kazanidis, I., \& Wells, D. (2018). Augmenting the learning experience in primary and secondary school education: a systematic review of recent trends in augmented reality game-based learning. Virtual Reality, 23(4), 329-346. https://doi. org/10.1007/s10055-018-0347-2

Pellas, N., Kazanidis, I., \& Palaigeorgiou, G. (2019). A systematic literature review of mixed reality environments in K-12 education. Education and Information Technologies, 25, 2481-2520. https://doi.org/10.1007/s10639-019-10076-4

Pelletier, K., Brown, M., Brooks, C., McCormack, M., Reeves, J., Arbino, N., Bozkurt, A., Crawfors, S., Czerniewicz, L., Gibson, R., Linder, K., Mason, J., \& Mondelli, V. (2021). EDUCAUSE Horizon Report. Teaching and Learning Edition. Educause.

Ponto, K., Kuester, F., Nideffer, R., \& Penny, S. (2006). Virtual bounds: A teleoperated mixed reality. Virtual Reality, 10(1), 41-47. https://doi.org/10.1007/s10055-006-0030-x 
Potkonjak V., Gardner M., Callaghan V., Mattila P., Guetl C., Petrović V. M., \& Jovanović K. (2016). Virtual Laboratories for Education in Science, Technology, and Engineering. Computers \& Education, 95, 309-327. https://doi.org/10.1016/j.compedu.2016.02.002

Prendes, M. P., \& Cerdán , F. (2021). Tecnologías avanzadas para afrontar el reto de la innovación educativa. RIED. Revista Iberoamericana de Educación a Distancia, 24(1), 35-53. https://doi.org/10.5944/ried.24.1.28415

Radianti, J., Majchrzak, T. A., Fromm, J., \& Wohlgennant, I. (2020). A systematic review of immersive virtual reality applications for higher education: Design elements, lessons learned, and research agenda. Computers \& Education, 147, 1-33. https://doi.org/10.1016/j. compedu.2019.103778

Ribeiro, R. (15 de noviembre de 2021). Metaverse and the educational potential: Is it so far away? Cambridge University Press. https://bit.ly/34PDBR0

Rodríguez, P. (2021). Metaverso: hay otros mundos, pero están en éste. Fundación Telefónica. https://bit.ly/3qEHvVe

Rogers, S. (2019). Virtual reality: The learning aid of the 21st century. Forbes

Schroeder, R. (1996). Possible Worlds: The Social Dynamic of Virtual Reality Technologies. Westview Press.

Siyaev, A., \& Jo, G. S. (2021). Towards aircraft maintenance metaverse using speech interactions with virtual objects in Mixed Reality. Sensors, 21(6), 1-21. https://doi.org/10.3390/ s21062066

Slater, M. (2003). A note on presence terminology. Presence Connect, 3(3), 1-5.

Smithson, A. (5 de enero de 2022). The Metaverse Manifesto. https://bit.ly/3tdHfyu

Sollervall, H. (2012). Collaborative mathematical inquiry with augmented reality. Research $\varepsilon$ Practice in Technology Enhanced Learning, 7(3), 153-173.

Stephenson, N. (1992). Snow Crash. Bantam Books.

Suh, A., \& Prophet, J. (2018). The state of immersive technology research: A literature analysis. Computers in Human Behavior, 86, 77-90. https://doi.org/10.1016/j.chb.2018.04.019

Tamura, H., Yamamoto, H., \& Katayama, A. (2001). Mixed reality: Future dreams seen at the border between real and virtual worlds. Computer Graphics and Applications, 21(6), 64-70. https://doi.org/10.1109/38.963462

Tang, Y. M., Au, K. M., Lau, H. C., Ho, G. T., \& Wu, C. H. (2020). Evaluating the effectiveness of learning design with mixed reality (MR) in higher education. Virtual Reality, 24(4), 797-807. https://doi.org/10.1007/s10055-020-00427-9

Trowler, V. (2010). Student engagement literature review. Lancaster University. The Higher Education Academy.

Tscholl, M., \& Lindgren, R. (2016). Designing for learning conversations: How parents support children's science learning within an immersive simulation. Science Education, 100(5), 877-902. https://doi.org/10.1002/sce.21228

Wang, J. (2001). Handshakes in cyberspace: Bringing the cultural differences through effective intercultural communication and collaboration. National Convention of the Association for Educational Communications and Technology, 513-519. 
Weng, C., Rathinasabapathi, A., Weng, A., \& Zagita, C. (2018). Mixed Reality in science education as a learning support: A revitalized science book. Journal of Educational Computing Research, 57(3), 777-807. https://doi.org/10.1177/0735633118757017

Yuen, S. C., \& Yuen, P. K. (2008). Mobile learning. In L. A. Tomei (Ed.), Encyclopedia of information technology curriculum integration (pp. 95-99). Idea Group.

Yusoff, R. C. M., Ibrahim, R., Zaman, H. B., \& Ahmad, A. (2011). Evaluation of user acceptance of mixed reality technology. Australasian Journal of Educational Technology, 27(8), 1369-1387. https://doi.org/10.14742/ajet.899

Zhan, T., Yin, K., Xiong, J., He, Z., \& Wu, S.T. (2020). Augmented reality and virtual reality displays: perspectives and challenges. IScience, 23(8), 1-13. https://doi.org/10.1016/j. isci. 2020.101397

Zheng, R.Z. (2020). Cognitive and affective perspectives on immersive technology in education. IGI Global. 